\title{
Cystoscopy Findings in Females with Recurrent Urinary Tract Infection
}

\author{
Azar Daneshpajooh, ${ }^{1, *}$ and Mahboubeh Mirzaei ${ }^{1}$ \\ ${ }^{1}$ Department of Urology, Clinical Research Center, Shahid Bahonar Hospital, Kerman University of Medical Sciences, Kerman, IR Iran \\ "Corresponding author: Azar Daneshpajooh, Fellowship of Female Urology, Department of Urology, Shahid Bahonar Hospital, Kerman University of Medical Sciences, Kerman, \\ IR Iran. Tel: +34-32235011, Fax: +34-32239188, E-mail: azdaneshpajooh@yahoo.com \\ Received 2018 February 11; Accepted 2018 May 07.
}

\begin{abstract}
Objectives: Recurrent urinary tract infection is common in adult females. This study aimed at identifying lower urinary tract abnormalities in females with recurrent urinary tract infections based on cystoscopy findings and evaluating the diagnostic yield of cystoscopy in these patients.

Methods: In this cross-sectional study, females (above 18 years) with recurrent urinary tract infection, who were referred to the urology clinic of Kerman University of Medical Sciences, Iran, in 2014 to 2016, were evaluated. After collecting demographic information, urine culture and urinary tract ultrasonography were performed for all patients; those with gross or microscopic hematuria, abnormality in imaging, neurogenic bladder, genitourinary cancer, and congenital urinary tract abnormality were excluded. Eligible patients underwent urethrocystoscopy, and the findings were recorded. Associations between clinical risk factors and abnormal findings were analyzed.

Results: Of the 88 patients (mean age of 48 years), who underwent urethrocystoscopy, urinary tract abnormalities were reported in 36 (40.9\%) patients, among whom 19 (21.5\%) and 17 (19.4\%) had clinically significant and non-significant abnormalities, respectively. Significant abnormalities included 14 cases of urethral stricture, one case of mesh erosion into the urethra, one case of periurethral gland abscess, two cases of bladder diverticulum, and one case of refluxing ureteral orifice.

Conclusions: Although the majority of urinary tract abnormalities among females with recurrent urinary tract infection could be identified via imaging modalities, yet, it seems that cystoscopy is an appropriate method for the diagnosis of urinary tract disorders in some cases, especially in patients, who are unresponsive to common treatment strategies.
\end{abstract}

Keywords: Urinary Tract Disorder, Recurrent Urinary Tract Infection, Cystoscopy

\section{Background}

Urinary Tract Infection (UTI) is one of the most common infections in females and is considered a challenge among physicians, imposing major costs on healthcare systems (1). Every year, almost $10 \%$ of females experience one episode of UTI, and 53\% above the age of 55 years and $36 \%$ of younger females report a recurrence within one year $(2,3)$. Recurrent UTI is symptomatic UTI that follows the resolution of an earlier episode after appropriate treatment and is defined as two or more infections during a sixmonth period or three or more infections in one year $(2,4$, $5)$. Recurrent UTI is quite common among females and has major impact on patient quality of life and can lead to sexual disorders and reduced social function (6).

Recurrent UTI is divided to two categories; "reinfection" and "bacterial persistence". Reinfection occurs at irregular intervals and is caused by different organisms out- side the urinary tract, while bacterial persistence occurs at regular intervals and is caused by the same organisms within the urinary tract (7-9).

The majority of rUTIs are believed to be reinfections and are much more common in females without urologic abnormality; in contrast, bacterial persistence may occur in females with structural abnormalities or with a foreign body in the urinary tract.

Diagnostic evaluations for rUTI include history-taking and physical examination, which involve assessing the patient for risk factors and confirming the presence of a bacterial UTI. Further investigations are generally unnecessary in patients with a history of uncomplicated lower rUTI, and imaging modalities and cystoscopy have a low diagnostic yield (10-13); however, a small number of females with rUTIs have abnormalities of the urinary tract, including urolithiasis, urethral stricture, diverticulum or a foreign body. In these patients, the causes of the rUTI should be found and 
corrected to potentially cure these patients (9); imaging modalities and invasive methods, such as cystoscopy, can also be helpful in these cases $(14,15)$.

Currently, non-invasive investigations, such as ultrasound are often performed to exclude abnormality of the urinary tract, yet the role of cystoscopy remains unclear.

Given the current lack of evidence and well-established guidelines regarding which females with rUTI warrant cystoscopy and considering the scarcity of research on the advantages of cystoscopy in females with recurrent UTI.

\section{Objectives}

The current researchers aimed at evaluating the role of cystoscopy in the determination of clinically significant pathology in females with rUTIs and the predictive value of risk factors in cystoscopy findings.

\section{Methods}

This cross-sectional study was performed on females older than 18 years old with a diagnosis of recurrent UTI, who were referred to the urology clinic of Kerman University of Medical Sciences, Kerman, Iran between 2014 and 2016. The inclusion criteria were as follows: 1) a history of at least two episodes of UTI over the past six months or at least three episodes of UTI in one year; 2) normal abdominopelvic imaging; and 3) a willingness to participate in the study. The exclusion criteria were as follows:1) hematuria; 2) neurogenic bladder; 3) genitourinary cancer; 4) congenital urinary tract abnormality; and 5) an unwillingness to continue the study for any reason.

A total of 88 eligible patients were recruited in the study via accessible sampling. Before the study, the research team fully explained the study objectives and methods to the participants and assured them about the confidentiality of the data. After obtaining consent forms from the patients, history taking and physical examinations were carried out. Demographic data and clinical risk factors were recorded. Complete urinalysis testing and urine culture were performed for all patients.

Cystoscopy was performed on patients under local anesthesia or sedation in the lithotomy position using a semi-rigid cystoscope (No. 17) with a $30^{\circ}$ lens. Urinary tract disorders that were evaluated using a cystoscope, included significant abnormalities, such as urethral stricture, bladder diverticulum, fistula, ulcers, stones, foreign bodies, ureteral orifice abnormality and abscesses, while non-significant abnormalities included bladder mucosal changes. Bladder biopsy was performed for patients with suspicious bladder mucosal lesions.
The data were analyzed using SPSS version 21. For statistical analysis, descriptive and analytical (Chi-square test, Fisher's exact test, and t-test) tests were performed.

\section{Results}

In this study, 88 eligible patients underwent cystoscopy. The mean age of the patients was 48.33 years (range: 18 to 70 years) and the mean Body Mass Index(BMI) was $25.7 \mathrm{~kg} / \mathrm{m}^{2}$; the mean age of the participants during the first episode of infection was 31 years (range: 10 to 42 years). Based on the findings, the mean frequency of UTI was five times per year. In total, $80 \%$ of the patients were married, and $54 \%$ did not use contraceptive methods; the mean parity among the participants was three. In addition, $22 \%$ of the patients had a history of pelvic organ prolapse, $37 \%$ had a history of pelvic surgeries, and $46 \%$ were menopausal (Table 1).

\begin{tabular}{|c|c|c|}
\hline Variable & Frequency & Percentage \\
\hline \multicolumn{3}{|l|}{ Marital status } \\
\hline Married & 80 & 90.9 \\
\hline Single & 8 & 9.1 \\
\hline \multicolumn{3}{|c|}{ Use of contraceptive methods } \\
\hline Yes & 34 & 38.6 \\
\hline No & 54 & 61.4 \\
\hline \multicolumn{3}{|c|}{$\begin{array}{l}\text { Types of prevention methods for affected } \\
\text { women }\end{array}$} \\
\hline Contraceptive pills & 26 & 76.4 \\
\hline Natural & 5 & 14.7 \\
\hline Condom & 3 & 8.9 \\
\hline \multicolumn{3}{|l|}{ Pelvic organ prolapse } \\
\hline Yes & 22 & 25 \\
\hline No & 66 & 75 \\
\hline \multicolumn{3}{|c|}{$\begin{array}{l}\text { History of prolapse surgery and } \\
\text { hysterectomy }\end{array}$} \\
\hline Yes & 37 & 42 \\
\hline No & 51 & 58 \\
\hline \multicolumn{3}{|l|}{ Menopause status } \\
\hline Yes & 46 & 52.2 \\
\hline No & 42 & 47.8 \\
\hline
\end{tabular}

In the present study, among 88 patients undergoing cystoscopy, 36 (40.9\%) had urinary tract abnormalities. In total, 19 (21.5\%) patients had significant abnormalities, while 17 (19.4\%) had clinically non-significant abnormalities. Significant abnormalities included 14 cases of ure- 
thral stricture, one case of mesh erosion in the urethra, one case of periurethral gland abscess, two cases of bladder diverticulum, and one case of refluxing ureteral orifice. Non-significant abnormalities included 17 cases of bladder mucosal change, of which 11 underwent bladder biopsy, including seven cases of squamous metaplasia, two cases of cystitis cystica, one case of chronic cystitis, and one case of cystitis glandularis. Other cases included trabeculation of the bladder mucosa (five cases) and bladder glomerulation (one case) (Table 2 ).

Table 2. Determination of Females Cystic Findings with Recurrent Urinary Tract Infections

\begin{tabular}{|c|c|c|}
\hline Cystoscopy Finding & Frequency & Percentage \\
\hline \multicolumn{3}{|l|}{ Non-specific findings } \\
\hline Bladder mucosal changes & 17 & 19.3 \\
\hline \multicolumn{3}{|l|}{ Proprietary findings } \\
\hline Stenosis & 14 & 15.9 \\
\hline Bladder diverticulum & 2 & 2.2 \\
\hline Pre urethral gland abscess & 1 & 1.1 \\
\hline Mesh erosion in urethral & 1 & 1.1 \\
\hline Ureteral changes (reflux) & 1 & 1.1 \\
\hline Urinary system stones & 0 & 0 \\
\hline Urinary system tumors & 0 & 0 \\
\hline Diverticulum urethral & 0 & 0 \\
\hline Urethral external object & 0 & 0 \\
\hline \multicolumn{3}{|l|}{ Biopsy } \\
\hline Yes & 11 & 12.5 \\
\hline No & 77 & 87.5 \\
\hline \multicolumn{3}{|l|}{ Types of biopsy findings } \\
\hline Squamous metaplasia & 7 & 63.6 \\
\hline Cystitis cystica & 2 & 18.1 \\
\hline Cystitis glendolaris & 1 & 9 \\
\hline Chronic cystitis & 1 & 9 \\
\hline
\end{tabular}

Evaluation of the relationship between risk factors and cystoscopy findings revealed that only the patient's age at the first episode of urinary infection was associated with the frequency of urethral stricture $(\mathrm{P}=0.008)$; mean age during the first episode of urinary infection was significantly higher in patients with urethral stricture compared to those without urethral stricture (Table 3). In addition, the findings revealed that cystoscopy findings were not related to parameters, such as BMI, age, marital status, the use of contraceptive methods, parity, pelvic organ prolapse, history of pelvic surgery, the frequency of UTI per year, or menopausal status in females with recurrent UTI $(\mathrm{P}>0.05)$.
Table 3. The Relationship Between Urethral Stenosis and the Age of the First Episode of Urinary Tract Infection in Females with Urinary Tract Infection

\begin{tabular}{cccc}
\hline Variable & Mean & Standard Deviation & PValue \\
\hline Urethral stenosis & & & 0.008 \\
Yes & 38.24 & 9.90 & \\
No & 30.43 & 10.48 & \\
\hline
\end{tabular}

\section{Discussion}

The purpose of this study was to determine the advantages of cystoscopy for the diagnosis of urinary tract disorders in females with recurrent UTI. Although the majority of urinary tract disorders could be identified via imaging examinations, some abnormalities could be diagnosed more efficiently via cystoscopy.

In the present study, among 88 patients undergoing cystoscopy, 19 (21.5\%) cases had significant abnormalities in cystoscopy, including 14 cases of urethral stricture, one case of mesh erosion in the urethra, one case of periurethral gland abscess, two cases of bladder diverticulum, and one case of refluxing ureteral orifice.

Lawrentschuk et al. retrospectively investigated the diagnostic yield of cystoscopy in 118 patients with recurrent UTI. Nine (8\%) females had clinically significant abnormalities, including urethral stricture (six), bladder calculus (one), bladder diverticulum (one), and colovesical fistula (one) (16).

Moreover, in the mentioned study, only one case was missed by imaging; therefore, when compared to cystoscopy, imaging had a high negative predictive value (99\%), indicating that imaging may be useful in contributing to a decision not to perform cystoscopy if the findings are normal. Additionally, the authors noted that the positive predictive value was low for risk factors in predicting cystoscopy findings and concluded that females without clinical risk factors for recurrent UTI and with normal imaging findings could omit cystoscopy. However, the authors mentioned that cystoscopy should be considered if the females were in a subgroup having clinical risk factors or abnormal imaging results.

In the current study, patients with recurrent UTI, who had normal imaging (ultrasonography) results were evaluated via cystoscopy; however, although the imaging results were normal, this research detected a significant abnormality in $21.5 \%$ of these patients, indicating that even when ultrasonography findings are normal, cystoscopy findings can be abnormal.

In another study by Howles et al., the diagnostic performance of flexible cystoscopy in patients with profound lower urinary tract symptoms, recurrent urinary tract infection, and pain were examined retrospectively (17). Thir- 
teen (11.5\%) out of 113 patients had significant cystoscopy findings, including bladder cancer (1), urethral stricture (9), and intravesical calculi (4). Clinically relevant abnormalities were found in $11.5 \%, 19.3 \%$, and $0 \%$ of patients with profound lower urinary tract symptoms, recurrent UTI, and pain, respectively. In cases with recurrent UTI (15 females and eight males), bladder calculi were diagnosed in two and urethral stricture in three male patients, and only one significant abnormality (urethral stricture) was diagnosed in the subgroup of females, resulting in a 6.7-\% diagnostic yield for this group.

In the mentioned study, cystoscopy was recommended for patients with profound lower urinary tract symptoms and recurrent UTI. In addition, the frequency of urinary tract disorders was significantly higher in males (17).

Additionally, malignancy was only reported in one patient with profound lower urinary tract symptoms. In comparison, no malignancy was reported in the current study, although three patients had pre-malignancy findings, including cystitis cystica (two cases) and cystitis glandularis (one case), which required further follow-ups; in fact, the authors would not have been able to diagnose these conditions without cystoscopy.

In a recent study, Pagano et al. reported the overall yield of cystoscopy in detecting clinically significant pathology when compared to imaging alone in females with recurrent UTI. A total of 163 patients underwent cystoscopy, among whom 133 were assessed via imaging modalities (renal/bladder ultrasound or abdominopelvic Computed Tomography (CT). Among the patients subjected to imaging, 21 significant abnormalities were noted in 18 patients (13.6\%). Overall, cystoscopy identified nine (5.5\%) significant clinical findings (non-specific findings, such as cystitis cystica were not considered positive findings); of these, only five (3.8\%) cases were uniquely identified by cystoscopy and were missed by imaging modalities (one case of bladder diverticulum, two cases of urethral stenosis, one case of a foreign body with intravesical suture material, and one case of carcinoma in situ) (18).

In that study, abdominopelvic imaging was recommended to all patients, and the findings were compared with cystoscopy results. In contrast, in the current study, only patients with normal imaging (ultrasound) were recruited, and patients with abnormal imaging were excluded from the study. Moreover, in the mentioned study, patients in the high-risk group (kidney transplant, immunodeficiency, previous urogenital surgeries, and urinary tract stones) were not associated with a higher risk of abnormal cystoscopy or a higher risk of abnormal imaging. Similarly, in the current study, the authors did not find an association between risk factors, such as pelvic organ prolapse or prior urogynecologic surgery and abnormal cystoscopy.

Some causes of recurrent UTI are curable, and the aim of evaluating females with bacterial persistence is to discover these correctable causes. In the present study, 17 of 36 patients with abnormal cystoscopy findings were fully recovered after treatment, showing the effectiveness of cystoscopy for the diagnosis and treatment of some patients with recurrent UTI.

Moreover, one patient, who had received different treatments for recurrent UTI without any improvement, was referred to the clinic of the current research. After confirming the erosion of sub-urethral mesh into the urethra, the patient underwent surgical treatment and was cured. This case demonstrates that some causes of infection are treatable in patients with complaints of recurrent UTI.

On the other hand, two patients had small bladder diverticula without the need for intervention, and 17 of 36 patients had non-specific cystoscopy findings, of whom 11 underwent bladder biopsy, including seven cases of squamous metaplasia, two cases of cystitis cystica, one case of chronic cystitis, and one case of cystitis glandularis. These patients did not need clinical intervention.

Considering the scarcity of cystoscopy findings, which are not reported in imaging assessments, determining the importance of cystoscopy in cases with recurrent UTI requires further analysis.

Although cystoscopy seems to cause discomfort, pain, hematuria, infection, and anesthesia-related complications in patients, cystoscopy could help discover some curable causes of recurrent UTI, especially in patients with atypical presentations, such as obstructive symptoms or urinary incontinence. The current researchers suggest that a large cohort study of cystoscopy be conducted on patients with recurrent UTI and that different risk factors are examined to define the predictive value of risk factors for abnormal findings in cystoscopy and to define when cystoscopy is necessary for recurrent UTI.

\subsection{Conclusions}

Although the majority of cystoscopy findings in recurrent UTI are normal, the diagnosis of some urinary tract disorders is not possible through common imaging procedures; therefore, cystoscopy can be effective in some cases of recurrent UTI. In particular, this modality can be useful for patients, who are referred to urological clinics due to persistent infections that do not respond to common forms of therapy or for patients with atypical presentation. Cystoscopy can demonstrate some curable causes of recurrent UTI and determine possible pre-malignancies or malignant lesions and guide patient follow-ups. 


\section{References}

1. Miller JL, Krieger JN. Urinary tract infections cranberry juice, underwear, and probiotics in the 21st century. Urol Clin North Am. 2002;29:695-9.

2. Aydin A, Ahmed K, Zaman I, Khan MS, Dasgupta P. Recurrent urinary tract infections in women. Int Urogynecol J. 2015;26(6):795-804. doi: 10.1007/s00192-014-2569-5. [PubMed: 25410372].

3. Foxman B, Barlow R, D'Arcy H. Urinary tract infection: Self-reported incidence and associated costs. Ann Epidemiol. 2000;10:509-15.

4. Hooton TM. Recurrent urinary tract infection in women. Int J Antimicrob Agent. 2001;17(4):259-68.

5. Epp A, Larochelle A, Lavatsis D. Recurrent urinary tract infection. J Obstet Gynaecol Can. 2010;32:1082-101.

6. Levin A. Recurrent urinary tract infection: new theories and an old remedy. An Intern Med.1998;128(4):333-6.

7. American College of O; Gynecologists. ACOG Practice Bulletin No. 91: Treatment of urinary tract infections in nonpregnant women. $\mathrm{Ob}$ stet Gynecol. 2008;111(3):785-94. doi: 10.1097/AOG.0b013e318169f6ef. [PubMed: 18310389].

8. Franco AV. Recurrent urinary tract infections. Best Pract Res Clin Obstet Gynaecol. 2005;19(6):861-73. doi: 10.1016/j.bpobgyn.2005.08.003. [PubMed: 16298166].

9. Nicolle LE, Ronald AR. Recurrent urinary tract infection in adult women: diagnosis and treatment. Infect Dis Clin North Am. 1987;1(4):793-806. [PubMed: 3333659].

10. van Haarst EP, van Andel G, Heldeweg EA. Evaluation of diagnostic workup in young women reffered for recurrent urinary tract infections. Urolog. 2001;57:1068-72.
11. Segal AJ, Amis EJ, Bigongiari LR, Bluth EI, Bush WJ, Choyke PL, et al. Recurrent lower urinary tract infections in women. American College of Radiology. ACR Appropriateness Criteria. Radiology. 2000;215 Suppl:671-6. [PubMed: 11037482].

12. Engel G, Schaeffer AJ, Grayhack JT. The role of excretory urography and cystoscopy in the evaluation and management of women with recurrent urinary tract infection. J Urol. 1980;123:190-1.

13. Nickel JC, Wilson J, Morales A, Heaton J.Value of urologic investigation in a targeted group of women with recurrent urinary tract infections. Can J Surg. 1991;34(6):591-4. [PubMed: 1747838].

14. Lane DR, Takhar SS. Diagnosis and management of urinary tract infection and pyelonephritis. Emerg Med Clin North Arm. 2011;29(3):539-52.

15. Kumar V, Patel HR, Nathan SM, Miller RA, Lawson AH. Do we need to perform cystoscopy on all adults attending urology centres as outpatients? Urol Int. 2004;73(3):198-200. discussion 200. doi: 10.1159/000080827. [PubMed: 15539836].

16. Lawrentschuk N, Ooi J, Pang A, Naidu KS, Bolton DM. Cystoscopy in women with recurrent urinary tract infection. Int $J$ Urol. 2006;13(4):350-3. doi: 10.1111/j.1442-2042.2006.01316.x. [PubMed: 16734849].

17. Howles S, Tempest H, Doolub G, Bryant RJ, Hamdy FC, Noble JG, et al. Flexible cystoscopy findings in patients investigated for profound lower urinary tract symptoms, recurrent urinary tract infection, and pain. J Endourol. 2012;26(11):1468-72. doi: 10.1089/end.2012.0139. [PubMed: 22612791].

18. Pagano MJ, Barbalat Y, Theofanides MC, Edokpolo L, James MB, Cooper KL. Diagnostic yield of cystoscopy in the evaluation of recurrent urinary tract infection in women. Neurourol Urodyn. 2017;36(3):692-6. doi: 10.1002/nau.22998. [PubMed: 26998700]. 